\title{
Sauerkraut: A Probiotic Superfood
}

\section{Ryan Orgeron II ${ }^{1 *}$, Angela Corbin ${ }^{2}$, Brigett Scott $^{3}$}

${ }^{1}$ Department of Allied Health Sciences, Dietetics Graduate; ${ }^{2}$ Department of Biological Sciences, Faculty of Microbiology; ${ }^{3}$ Department of Allied Health Sciences, Faculty of Dietetics. Nicholls State University, Thibodaux, Louisiana 70301, USA

*Corresponding author: Ryan Paul Orgeron II, B.S. CPT, Department of Allied Health Sciences/ Dietetics, Nicholls State University, 906 E 1st St, Thibodaux, LA, 70310 USA

Submission Date: May 9, 2016; Acceptance Date: August 27, 2016; Publication Date: August 30,2016

\begin{abstract}
Background: Sauerkraut could potentially be a probiotic superfood. Sauerkraut does not require the use of a starter culture to cultivate these beneficial bacteria. All that is needed is a measure of salt and the cabbage. Naturally made, unprocessed sauerkraut contains probiotic microorganisms called Lactic Acid Bacteria (LAB). LAB is one of the most significant organisms and has established benefits. For example, Lactic Acid Bacteria has established benefits with the treatment of diarrhea, constipation, irritable bowel syndrome, infections (urogenital, urinary and candida). LAB have also been shown to enhance immune system function to help prevent various illnesses and promote lactose digestion. Nevertheless, there is very little conclusive evidence on how much LAB is in sauerkraut and if there is enough present to confer benefits.
\end{abstract}

Purpose: Determine if various serving sizes of homemade sauerkraut ( 2 Tbsp., $1 / 2$ cup, \& 1 cup) meets the recommended CFU range in comparison to a control (supplement probiotic).

Methods: Sauerkraut was prepared with no starter culture. Modified Lactobacillus Media was used to culture LAB. The capsule serving $(0.8 \mathrm{~g})$ was diluted with $99.2 \mathrm{~g}$ PBS (1/100) and $10 \mathrm{~g}$ of sauerkraut was diluted with $90 \mathrm{~g}$ PBS (1/10). For serial dilutions, $1 \mathrm{~mL}$ of each of the samples was diluted into $9 \mathrm{~mL}$ MRS broth for the dilutions $\left(10^{-1}-10^{-10}\right)$. Then $1 \mathrm{~mL}$ from each test tube is transferred into corresponding petri dishes. Melted Lactobacillus MRS agar is then mixed into each of the petri dishes. All petri dishes are then placed into a candle light jar and placed in an incubator at 37 degrees Celsius $\left(98.6^{\circ} \mathrm{F}\right)$ for 5 days. After five days, the petri dishes with 25-250 visible cultures were counted. The number counted was multiplied by the dilution which gave the total number CFUs/g. The LAB concentration of the sauerkraut was calculated by multiplying the $\mathrm{CFU} / \mathrm{g}$ times the serving size. 
Results: The results were calculated as the mean of CFUs recorded from the 3 trials for both the control (supplement) and the sauerkraut. The control group was calculated as a mean of 4.3x107 CFUs with a Relative Standard Deviation (RSD) of 3.5\% CFUs. The sauerkraut averages were calculated for each of the serving sizes. The 2 Tbsp. serving has a mean of $1.5 \times 106$ (RSD= $32 \%)$, the $1 / 2$ cup has a mean of $5.9 \times 106(\mathrm{RSD}=31 \%)$, and the 1 cup serving has a mean of $1.2 \times 107(\mathrm{RSD}=30 \%)$.

Conclusion: The study concludes that sauerkraut (even the two Tbsp. serving) meets the recommended CFU range. Based on these findings, sauerkraut can be considered a "probiotic superfood".

Keywords: Sauerkraut, probiotic superfood, supplement probiotic, Lactobacillus

\section{BACKGROUND}

Sauerkraut could possibly be one of most underrated superfoods. Sauerkraut is a name given to the end product of fermenting cabbage. One of the reasons sauerkraut is considered a superfood is because the fermentation makes all of the nutrients of the food more bioavailable than in its raw form. This means that all the vitamins, mineral, and phytonutrients that cabbage offers could become more bioavailable after fermentation. For example, Dr. Natasha Campbell-McBride states in her book "The amount of bioavailable vitamin C in sauerkraut is 20 times higher than in the same serving as raw cabbage" [1]. In addition to increased bioavailability, what really sets sauerkraut apart from most foods is its probiotic content. The main reason sauerkraut is a wonderful food is because it is full of probiotics. Probiotics are defined as living organisms which upon ingestion in certain numbers confer health benefits upon the host [2].

The gut microbiota has become one of the most popular topics in the industries of health and nutrition. Many studies have been done which demonstrate the many benefits that a healthy human gut (and all of the bacteria involved) can have on human health. The microbiota plays a major role in health and disease in humans and it is sometimes referred to as our "forgotten organ" [3]. Even more importantly, the gut microbiota interacts with the immune system by providing signals to promote the maturation of immune cells and the normal development of immune functions [4].

One of the most significant groups of probiotic organisms are the lactic acid bacteria (LAB) which were observed further in this study. Lactic Acid bacteria have established benefits such as the treatment of diarrhea, constipation, irritable bowel syndrome, and infections such as urogenital, urinary and candida. LAB have also been shown to enhance immune system function to help prevent various illnesses and promote lactose digestion. Some studies even show that these bacteria could even prevent certain cancers. While nearly all research confines LAB to dairy products like yogurt, sauerkraut stands out as a vegetable that also produces LAB [5]. Unlike yogurt, a starter culture is not needed for sauerkraut because the cabbages grown in healthy soil have all the bacteria they need to start fermentation. All that is added is salt which inhibits the growth of undesirable microorganisms but favors the growth of desired bacteria. 


\section{INTRODUCTION}

When it comes to sauerkraut, original studies dating back 1969 only found four strains of LAB[6]. A more recent study done in 2009 (with technique and technology advances), concluded that 15 strains of LAB with high conjugated linoleic acid (CLA)-producing ability were isolated from sauerkraut [7]. It should also be noted that within different stages of fermentation, there will be different organisms present. There is no definite identity or count on the LAB in sauerkraut. The topic of probiotics has become more popular to not only science but also to consumers as well. This becomes evident when considering the sales of these probiotic supplements. According to the Statista Statistic Portal, global probiotic sales are expected to jump from $\$ 25$ billion in 2011 to $\$ 42$ billion in 2016 [8]. This statistic shows that consumers are paying for these probiotics that are man-made in a lab and sold for a profit. There is nothing wrong with supplementation because there are many proven and effective probiotics, but the option of using fermented foods may be more efficient. The argument for sauerkraut (and other fermented foods) is that the families of strains that naturally culture together in fermented sauerkraut are more beneficial than the isolated strains found in supplements. In other words, probiotic therapy is based on the belief that certain strains are vital for our health, which leads to people going out and buying these isolated supplements. In reality, some research shows the genetic fluidity of bacteria suggests that variety and diversity may be more beneficial than specific strains [9]. In addition to the beneficial bacteria they produce, fermented foods also supply nutrition that no other sources can provide. The FDA recommends that quantitative composition of LAB CFUs to be in $10^{6}-10^{10}$ range[10]. Research also suggests that in order to confer benefits, an effective dose of probiotics falls within the $10^{6}-10^{8}$ range [11]. The question remains - can the natural probiotics found in sauerkraut be as beneficial as other options? An observation was made to determine if various serving sizes of homemade sauerkraut ( 2 Tbsp., $1 / 2$ cup, \& 1 cup) meets the recommended CFU range in comparison to a control (supplement probiotic).

\section{METHODS}

In order to determine how many LAB are in both the sauerkraut and the daily dose of a supplement, the first priority was to create a media (broth) and an agar (solidifying agent) for the bacteria to thrive in so they could be counted. The media recipe used was the Lactobacillus MRS Broth. This particular media was based on a media that was formulated and tested in 1960 and has been the placeholder for LAB cultivation media ever since [12]. The recipe was modified to use ingredients already available to keep costs minimal. The ingredient triammonium citrate was substituted with sodium citrate. The recipe used for the agar was the Lactobacillus MRS Agar, which is also based on the formulations and findings of the same study from 1960 [12]. Both the media and the agar were calculated and mixed to yield three times the amount of the original recipe. These media and agar were chosen together because of their efficient correspondence in previous studies. Both were stored in the refrigerator until use.

After the medium was created, the decision was made to use $10^{-10}$ dilutions for the trials. Dilutions were required to count bacteria because some plates could contain billions of bacteria which could not be manually counted. To increase accuracy, it is recommended to dilute and count the bacteria with lower counts. In particular, according to the FDA the recommendation is 
to count plates in the 25-250 range[13]. In doing so, if either of the test subjects surpasses the billion counts, it could still be calculated via dilution. Therefore, since $10\left(10^{-1}-10^{-10}\right)$ dilutions required the use of 10 test tubes for each subject for each trial (20 test tubes for a trial). Since three trials were conducted, 60 test tubes were utilized. In each of these test tubes would be $9 \mathrm{ml}$ of the Lactobacillus MRS Media. These media tubes were all kept refrigerated until use.

A trial began with the initial dilutions of the each test subject being blended for 90 seconds. The dilutions of both were a total of a $100 \mathrm{~g}$ dilution. Blenders were placed on a scale and touted before ingredients were added. The sauerkraut dilution consisted of $10 \mathrm{~g}$ of sauerkraut and $90 \mathrm{~g}$ of phosphate buffered saline. The $10 \mathrm{~g}$ amount of sauerkraut came from the $10 \mathrm{~g}$ being $1 / 10$ th of the $100 \mathrm{~g}$ dilution making the first dilution $\left(10^{-1}\right)$. The sauerkraut was taken out of its jar with sanitized tweezers (sanitized with alcohol and a flame). In a separate blender, the supplement dilution was the $0.2 \mathrm{~g}$ supplement capsule with the remaining $99.8 \mathrm{~g}$ being phosphate buffered saline making a $1 / 100^{\text {th }}$ dilution $\left(10^{-2}\right)$. Both dilutions were separately blended on high speed for 90 seconds.

Figure 1. Serial dilutions of sauerkraut and supplement samples

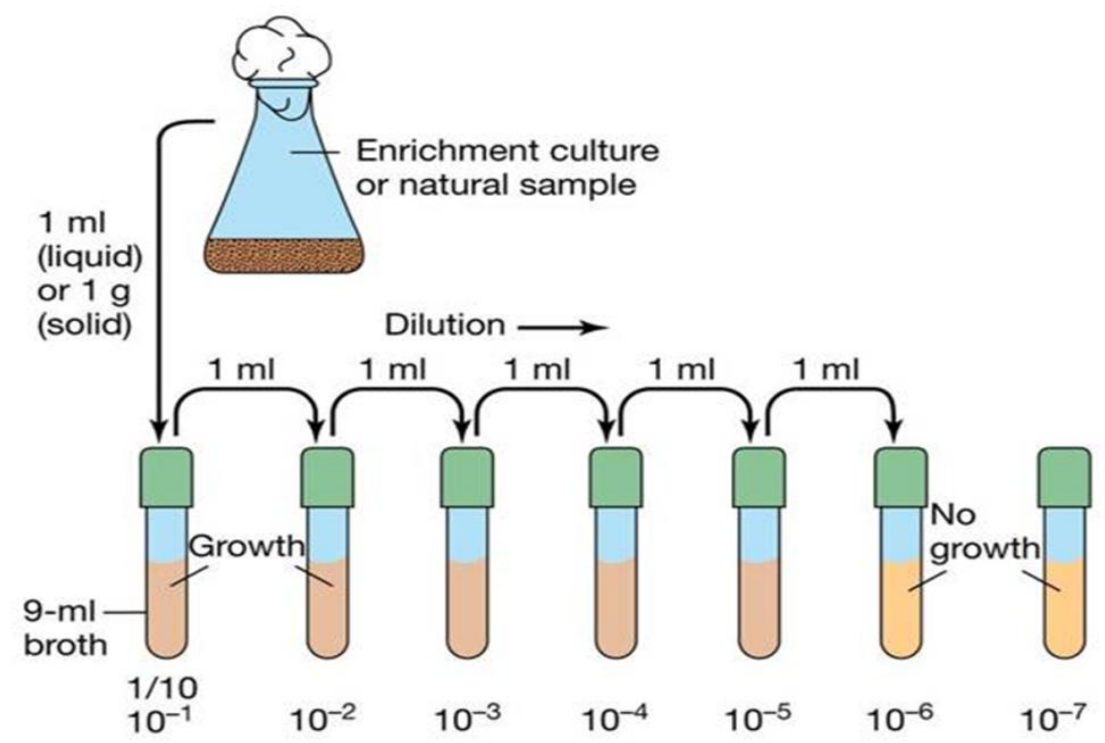

After the first dilutions were made, the twenty $9 \mathrm{ml}$ test tubes of media were taken out of refrigeration for the trial. The dilution of $1 \mathrm{ml}$ was transferred to the test tube via pipette set at 1 ml. Starting with the sauerkraut dilution, $1 \mathrm{ml}$ of the blended dilution was transferred into the first test tube which was then $10^{-2}$ dilution. After a 10 second vortex to stir the $10^{-2}$ test tube, $1 \mathrm{ml}$ was then taken from that test tube and transferred into the next test tube which was then labeled the $10^{-3}$ dilution. After each transfer, a new pipette tip was used to prevent previous bacteria from being carried into the next dilution. The process continued until the last $10^{-10}$ test tube received 1 $\mathrm{ml}$ of the previous $10^{-9}$ test tube. After all dilutions of the sauerkraut were done, the same procedure was conducted with the supplement dilution (Figure 1).

After diluting, the next step was to plate each of the dilutions. The first step of plate dilutions was to take out 10 petri dishes for each subject ( 20 per trial). The plates were then labeled $10^{-1}-10^{-10}$. After labeling, $1 \mathrm{ml}$ of each test dilution was then transferred into the 
corresponding petri dish starting with the most diluted then working towards the least. For example, $1 \mathrm{ml}$ was drawn out of the last $10^{-10}$ test tube and transferred into the petri dish labeled $10^{-10}$. The following was done all the way down to $10^{-1}$ in which $1 \mathrm{ml}$ of the initial blended dilution was then transferred into the petri dish with $10^{-1}$ label. Figure 2 shows all of the petri dishes after plate dilutions were complete. After $1 \mathrm{ml}$ of each test tube dilution was plated, the same procedure was followed for the other subject.

After all 10 dilutions are plated for both test subjects; the Lactobacillus MRS Agar was added to each of the petri dishes. The agar solidified at room temperature and was placed in the refrigerated storage, so it was heated in a microwave for two-four minutes, depending on size of container. The agar was brought to a boil and then set to cool (or cooled quicker in a cold water bath). Once in its liquid state, agar was poured into every plate along with the dilution. Agar was poured (enough to layer the bottom of the petri dish) and swirled three times one way and three times the opposite way to mix the dilution and the agar well. The agar solidified within one-three minutes in the petri dish. After all agar/dilution mixes solidified in the plate, another thin layer of agar is poured on top.

Once all petri dishes solidified, the next step was to use a candle light jar. All plates were placed inverted in the candle light jar. The candle is then light before the jar is closed and ready for incubation. The candle light jar holds up to 5-10\% CO2 which forms ideal conditions for bacteria to thrive. The whole candle light jar was then stored in incubation at 37 degrees Celsius (98.6 degrees Fahrenheit), which is the temperature of the human body. After 5 days of incubation, the bacteria were counted.

Figure 2. Sauerkraut plate dilution comparisons

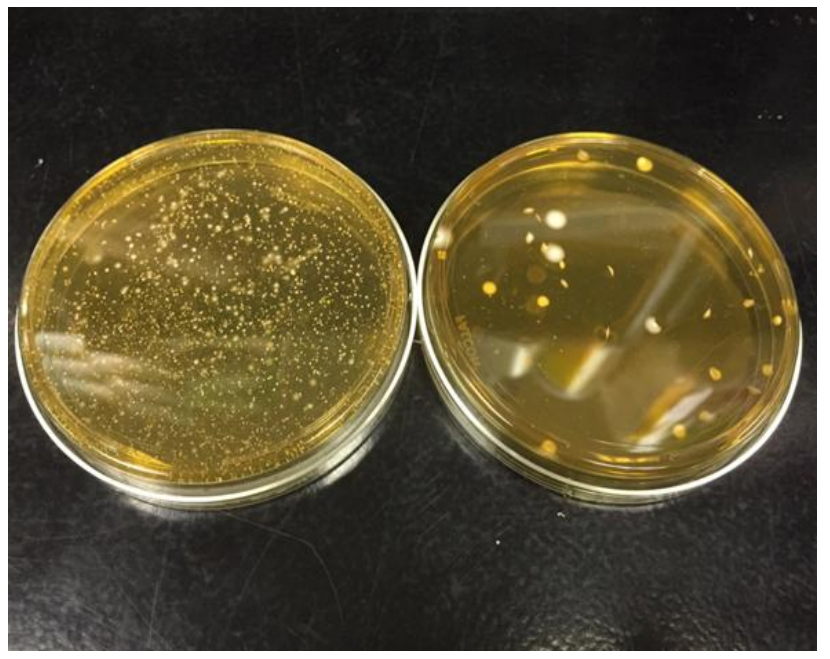

Counting the bacteria was done with a magnifying glass and a sharpie. The plates with 25250 visible colonies were placed under the magnifying glass. Figure 2 shows a plate not within a countable range (left) in comparison to a plate with 25-250 visible and countable colonies (right). As the colonies were counted they were dotted with the sharpie so they would not be recounted. After they were counted, the dilution number $\left(10^{-1}-10^{-10}\right)$ was then used to determine how many CFUs (colony forming units) were present. ${ }^{13}$ For example, if there were 45 colonies in the $10^{-3}$ petri dish, then it was read $4.5 \times 10^{4}$ CFUs. That would equal 45,000 CFUs. 
The sauerkraut serving sizes being calculated were $30 \mathrm{~g}, 118 \mathrm{~g}$, and $236 \mathrm{~g}$, and the amount tested was $10 \mathrm{~g}$ (for resource sparing). Therefore, the serving sizes being measured were divided by $10 \mathrm{~g}$ and then multiply that number by the CFUs of the 10 gram sample to get a total count for each serving size.

\section{RESULTS}

The results were calculated as the mean of CFUs recorded from the three trials for both the control (supplement) and the sauerkraut. The control group was calculated as a mean of $4.3 \times 10^{7}$ CFUs with a Relative Standard Deviation (RSD) of 3.5\%. The sauerkraut averages were calculated for each of the serving sizes. The 2 Tbsp. serving has a mean of $1.5 \times 10^{6}(\mathrm{RSD}=32 \%)$, the $1 / 2$ cup has a mean of $5.9 \times 10^{6}(\mathrm{RSD}=31 \%)$, and the 1 cup serving has a mean of $1.2 \times 10^{7}$ $(\mathrm{RSD}=30 \%)$.

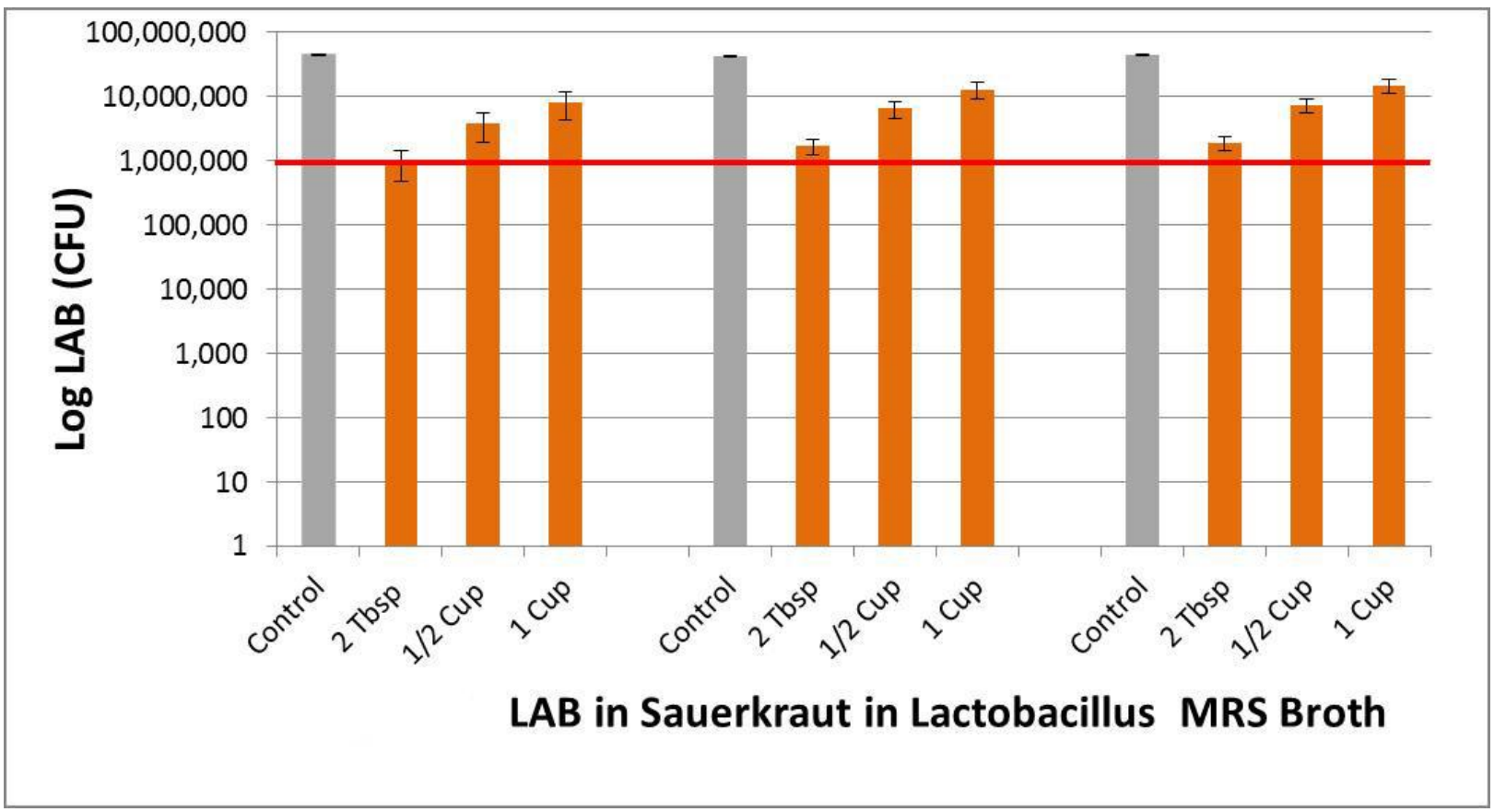

Figure 3. LAB in sauerkraut in Lactobacillus MRS broth

\section{DISCUSSION}

Initially, the research was going to compare store bought, shelf stable sauerkraut to homemade sauerkraut. After experimentation, it was concluded that store bought, shelf stable sauerkraut had little to no bacteria due to pasteurization and processing. An important note is that each trial was conducted on a different batch of sauerkraut. The first trial was conducted on a batch of six month (estimated time) old home fermented sauerkraut made by a third party (Dr. David Schultz). The second and third trial was conducted on batches made by the author, Ryan Orgeron II, and tested after three week ( 21 day) fermentation. It can be hypothesized that the first trial had a slightly lower count due to the age of the sample. The results show that all three trials display consistent results along with a consistent control throughout the experiment. The results conclude that homemade sauerkraut can supply an efficient amount of LAB to promote health 
benefits in small ( 2 tbsp.) and large (1 cup) serving sizes. Sauerkraut belongs to a rare category of foods which not only provide probiotics, but also nutrients of the cabbage in a more digestible state. The aim of the study was to culture and count strictly the LAB in sauerkraut, but it is expected that sauerkraut can be home to more microorganism species than which is currently known.

\section{CONCLUSION}

When compared to the recommended effective amount CFUs, the results indicated that sauerkraut did supply enough LAB to meet the standards set by research and the FDA. Interestingly, in two of the three trials, even the 2 tbsp. serving of sauerkraut were within the range. Based on these findings, sauerkraut should be considered a "Probiotic Superfood".

Competing interests: The authors declare that they have no competing interests with anyone or with any organizations.

Authors' Contributions: All authors contributed to this study. Ryan Orgeron II designed the research concept, conducted the study, performed the data analysis, collected literature/data for the research, and drafted/edited the manuscript. Angela Corbin supervised the entire study and initiated the methods of the study. Brigett Scott supervised the writing of the manuscript and corevised the manuscript.

Acknowledgments: Thank you to Dr. David Schultz for donating a third party sauerkraut sample for the first trial.

\section{REFERENCES}

1. Campbell-McBride N. Gut And Psychology Syndrome. [Cambridge, U.K.: Medinform Pub.]; 2010.

2. Food and Agriculture Organization of the United Nations and World Health Organization. Health and nutritional properties of probiotics in food, including powder milk with live lactic acid bacteria (World Health Organization Web site) . http://www.who.int/foodsafety/publications/fs_management/en/probiotics.pdf. (Accessed September 21,2015)

3. O'Hara AM, Shanahan F. The gut flora as a forgotten organ. EMBO Rep. 2006;7:688-93

4. J. Chow, S.M. Lee, Y. Shen, A. Khosravi, S.K. Mazmanian. Host-bacterial symbiosis in health and disease. Adv. Immunol., 107 (2010), pp. 243-274

5. Douglas, L.C. and Sanders, M.E. Probiotics and prebiotics in dietetic practice. J Am Diet Assoc. 2008; 108: 510-521

6. Pederson, C. S., and M. N. Albury. 1969. The sauerkraut fermentation. Technical bulletin 824. New York State Agricultural Experiment Station, Geneva, NY.

7. Zeng Z., Lin J., Gong D. Identification of lactic acid bacterial strains with high conjugated linoleic acid-producing ability from natural sauerkraut fermentations. J. Food Sci. 2009;74:154-158. 
8. Statista. Probiotic products' sales worldwide, 2015 | Statistic. 2015. Available at: http://www.statista.com/statistics/252930/probiotic-products-sales-worldwide/. Accessed October 21, 2015.

9. Smillie C. S., Smith M. B., Friedman J., Cordero O. X., David L. A., Alm E. J. (2011). Ecology drives a global network of gene exchange connecting the human microbiome. Nature 480: 241-244

10. Generally Recognized As Safe Notice; Lactobacillus Acidophilus. U.S. Food and Drug Administration: http://www.fda.gov/ucm/groups/fdagov-public/@fdagov-foods-gen/ documents/document/ucm266351.pdf. Accessed September 3, 2015

11. Minelli, E.B. and Benini, A., 2008. Relationship between number of bacteria and their probiotic effects. Microbiology and Ecology in Health and Disease 20: 180-183

12. de Man J. C., Rogosa M. and Sharpe M. Elisabeth. A medium for the cultivation of lactobacilli. Journal of Applied Microbiology.1960; vol. 23: 130-135

13. Maturin, L., \& Peeler, J. T. (2001, January). BAM: Aerobic Plate Count. Retrieved September 4, 2015, from

http://www.fda.gov/Food/FoodScienceResearch/LaboratoryMethods/ucm063346.htm 